\title{
The populations of hard X-ray and $\gamma$-ray sources: a correlation study and new possible identifications
}

\author{
A. Maselli ${ }^{1}$, G. Cusumano ${ }^{1}$, E. Massaro ${ }^{2}$, A. Segreto ${ }^{1}$, V. La Parola ${ }^{1}$, A. Tramacere ${ }^{3}$, and I. Donnarumma ${ }^{4}$ \\ 1 INAF-IASF Palermo, via U. La Malfa 153, 90146 Palermo, Italy \\ e-mail: maselli@ifc.inaf.it \\ 2 Dipartimento di Fisica, Università La Sapienza, Piazzale A. Moro 2, 00185 Roma, Italy \\ 3 INTEGRAL Science Data Centre, 1290 Versoix, Switzerland \\ ${ }^{4}$ INAF-IASF Roma, via Fosso del Cavaliere 100, 00133 Roma, Italy
}

Received 18 March 2011 / Accepted 9 April 2011

\section{ABSTRACT}

\begin{abstract}
Aims. We present the results of our analysis devoted to researching Galactic and extragalactic sources emitting in the energy bands surveyed by both the Swift-BAT and the Fermi-LAT telescopes.

Methods. We cross-correlated the Fermi-LAT 1-year point source catalogue (1FGL) of gamma-ray sources and the second Palermo BAT catalogue (2PBC) of hard X-ray sources, establishing a correspondence between sources when their error boxes overlap. With dedicated software for reducing BAT data, we also extracted the value of the detection significance $\sigma$ in the BAT 15-150 keV all-sky map in the direction of the 1FGL sources and took those above the threshold $\sigma_{\mathrm{T}}^{\star}=3$ into account.

Results. We obtain 62 firm correspondences by cross-correlating the 1FGL and 2PBC catalogues and verifying the agreement between the identified counterparts. The number of objects emitting in both the hard X-ray and the $\gamma$-ray energy bands rises to $104(\sim 7 \%$ of all the 1FGL objects) when considering all the sources with a detection significance down to $\sigma_{\mathrm{T}}^{\star}$. This group of objects is made of 82 extragalactic sources, the largest number of which are blazars, 15 Galactic sources, and seven unidentified objects. We discuss the blazar content of the collected 1FGL-2PBC sources and compare their redshift distribution with that of the whole blazar population as reported in the second edition of the BZCAT blazar catalogue. We describe in greater detail the properties of two unidentified sources at low Galactic latitude, 1FGL J0137.8+5814 and 1FGL J2056.7+4938, and support their classification as blazars after the analysis of their broad-band spectral energy distribution.
\end{abstract}

Key words. galaxies: active - BL Lacertae objects: general - radiation mechanisms: non-thermal

\section{Introduction}

The present generation of space observatories for high-energy astrophysics is characterised by large area and wide-field instrumentation. This is the case for the Burst Alert Telescope (BAT, Barthelmy et al. 2005) and the Large Area Telescope (LAT, Atwood et al. 2009) onboard Swift and Fermi-GST, respectively. One of the main throughputs of these instruments is the discovery of a thousand hard X-ray and $\gamma$-ray sources and the possibility of performing investigations into the population properties and evolution that are much more accurate than in the past.

The first catalogue of $\gamma$-ray point sources (1FGL, Abdo et al. 2010a), based on data obtained in the 11 months after the beginning of scientific operation (2008 August 4) contains 1451 entries, and a fraction of about $40 \%$ of them do not have reliable counterparts. A rather similar situation is also occurring with the hard X-ray sources detected by BAT: the second Palermo BAT catalogue (Cusumano et al. 2010b, hereafter $2 \mathrm{PBC}$ ), which covers the observation period from November 2004 to May 2009 and is the richest one in this energy range, also includes 177 unassociated sources, over a total number of 1256. A multifrequency approach based on the crosscorrelation of catalogues in different energy bands from the radio to the $\gamma$ rays can be very useful, if not essential, for unravelling the nature of unidentified sources.

In this paper we use the $1 \mathrm{FGL}$ and the $2 \mathrm{PBC}$ catalogues to compare the populations of hard X-ray and $\gamma$-ray sources and to search for possible correspondences among them. The paper is organised as follows. We report the details of the cross-correlation between the two catalogues in Sect. 2 and of the cross-correlation of the 1FGL catalogue with the BAT $15-150 \mathrm{keV}$ all-sky map of the detection significance $\sigma$ in Sect. 3. We discuss our results in Sect. 4 giving particular emphasis to the blazar content of the collected group of sources. In Sect. 5 we describe the properties of two unidentified objects in the 1FGL catalogue at low Galactic latitude, with significant emission in the hard X-ray band. We have collected all the available data in the literature and analyse their broad band spectral energy distribution (SED) in detail to support their classification as blazars. The main results of our analysis are summarised in Sect. 6.

\section{Correspondences between 1FGL and $2 \mathrm{PBC}$ sources}

The 2PBC catalogue was obtained from the reduction of the BAT data collected over the 54 months since the launch of the Swift mission using the dedicated software BATIMAGER (Segreto et al. 2010). It contains 1256 hard X-ray sources detected at a significance level higher than $\sigma_{\mathrm{T}}=4.8$, and their coordinates are given with a 95\% confidence level radius $r_{\text {BAT }}$. A counterpart was associated to 1079 sources $(\sim 86 \%)$; for 26 of them, a double association was found, and in two cases three 


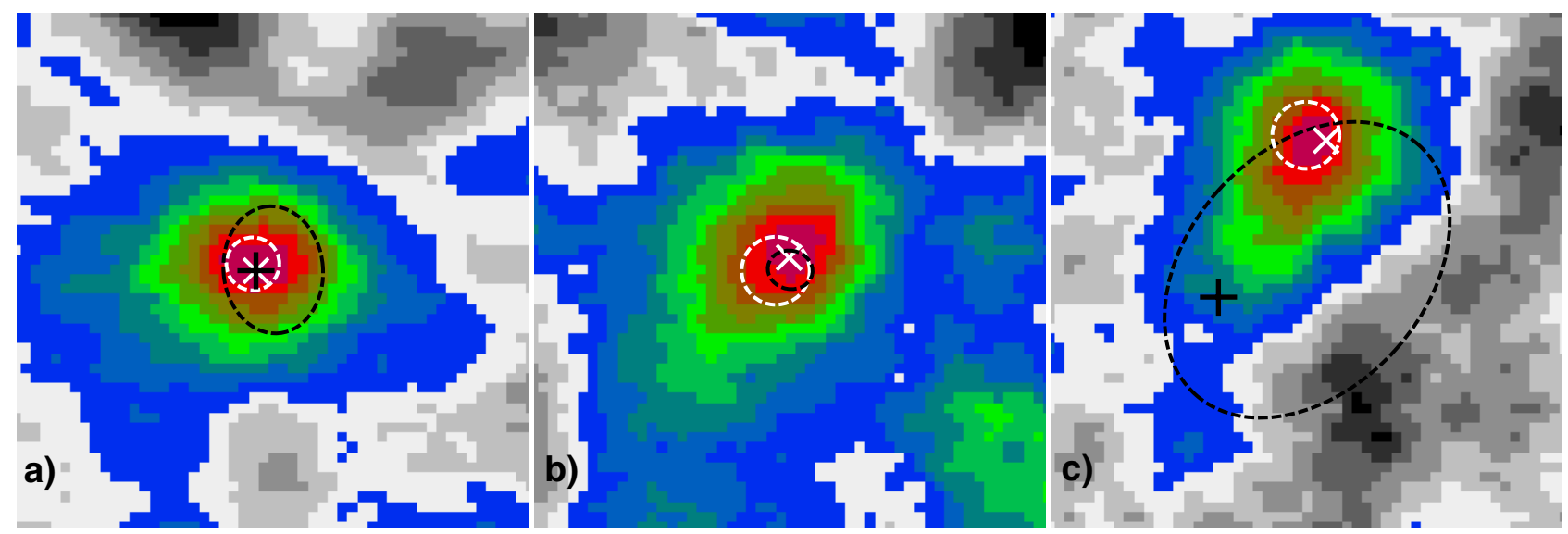

Fig. 1. Details of the BAT 15-150 keV all-sky map of the detection significance $\sigma$ at the position of a) 1FGL J1103.7-2329 (left panel), b) 1FGL J2056.7+4938 (middle panel), and c) 1FGL J0238.3-6132 (right panel). A dashed line represents the position of the 1FGL (black ellipse) and the 2PBC (white circle) sources with its uncertainty. For each source, the position of the associated counterpart is indicated by a cross of the corresponding colour. Each square map is $\sim 60^{\prime} \times 60^{\prime}$; the colour scale is optimised for each hard X-ray source.

possible counterparts were proposed. There were 177 sources without any associated counterpart.

We matched the $2 \mathrm{PBC}$ catalogue with the 1FGL catalogue to search for sources emitting in both the energy bands surveyed by the Swift-BAT and the Fermi-LAT telescopes. The positions of the 1451 1FGL $\gamma$-ray sources, determined by means of a maximum likelihood algorithm, are given with an uncertainty ellipse corresponding to the $95 \%$ probability of locating the source, and 58 objects are reported without any positional uncertainty. We adopted, when available, the mean value of the two axes of the $95 \%$ confidence ellipse as the radius $r_{\text {LAT }}$ of the error circle for a 1FGL source. We calculated the angular distance $d$ among the centroids of 1FGL and 2PBC sources and established a correspondence when the two error circles overlap, adopting the condition $d \leq\left(r_{\mathrm{BAT}}+r_{\mathrm{LAT}}\right)$. After applying this criterion we obtain 77 correspondences. We define a simple parameter to estimate the quality of the correspondence and use the expression $Q=d / r_{\mathrm{BL}}$, where $r_{\mathrm{BL}}$ is the higher value between $r_{\mathrm{BAT}}$ and $r_{\mathrm{LAT}}$. The parameter $Q<1$ implies that the wider error circle includes both centroids. This value occurs for 64 correspondences, while in four cases we find $Q>1.4$.

As a further step, we consider the possibility that the choice of the mean value of the axes of the $95 \%$ uncertainty ellipse, adopted to estimate the 1FGL uncertainty region, may be not firmly reliable. This aspect arises particularly in cases of high eccentricity values, in which the orientation of the 1FGL ellipse with respect to the $2 \mathrm{PBC}$ circle must be taken into account. To gain confidence in the established correspondence between sources, we computed the axial ratio of the uncertainty ellipse and verify in the sky map the 25 cases $(\sim 1 / 3)$ in which we find values higher than 1.2. In all but four cases, not only is the centroid of the 2PBC source inside the 1FGL ellipse, but also the associated counterpart.

Further correspondences have been established for nine of the 58 1FGL sources with no positional uncertainty, since the position of the 1FGL source is within the error circle of the 2PBC source. The remaining 49 cases were all unambiguously discarded, because no $2 \mathrm{PBC}$ source was found in the proximity of these 1FGL sources. The final list includes 86 1FGL2PBC correspondences for a total of 84 1FGL sources: two of them in fact have a possible correspondence with two different $2 \mathrm{PBC}$ sources. This confusion is due to crowded fields, in one case close to the Galactic centre direction. Moreover, we find a correspondence in which two possible counterparts, both high-mass X-ray binaries, have been associated to the same 2PBC source in the Small Magellanic Cloud. The list of correspondences has been split in two parts reported in Tables 1 and 2 according to the Galactic latitude, with 63 correspondences at $|b|>10^{\circ}$ and 23 at $|b|<10^{\circ}$. For each correspondence, we report the 1FGL and $2 \mathrm{PBC}$ identifiers, the associated counterparts, and their classification. For a few AGNs the counterpart, missing in the 1FGL catalogue, has been found in the first catalogue of active galactic nuclei detected by the Fermi Large Area Telescope (Abdo et al. 2010b), and these cases are marked with an asterisk $\left(^{*}\right)$ in Table 1 . The values of the $Q$ parameter and the agreement between the corresponding counterparts are reported in the last two columns. We mark with a colon the $Q$ values of the four correspondences for which the $2 \mathrm{PBC}$ counterparts are outside the 1FGL uncertainty ellipse. In 62 cases (marked with " $y$ ") the counterpart is the same, while in 11 cases (marked with " $n$ ") the counterparts associated with the high-energy sources are different: in these cases the correspondence is supposed to be due to chance. We also find 13 correspondences for which both sources, or just one of them, lack the associated counterpart. Assuming that the 1FGL-2PBC correspondence is the result of high-energy emission from a single source, we can tentatively associate a counterpart to these 1FGL sources. All these correspondences, with only one exception, are characterised by a value of the $Q$ parameter lower than unity.

We plot in Fig. 1 the details of the BAT 15-150 keV all-sky map of the detection significance $\sigma$ for some of these correspondences. In the left hand panel of Fig. 1 we report the case in which the 1FGL and the 2PBC sources correspond to the same object, the BL Lac 1H 1100-230. In the middle panel we report the case of the source 1FGL J2056.7+4938 that has not been identified in the 1FGL catalogue. The correspondence with the 2PBC source suggests the X-ray source RXJ2056.6+4940 as a possible counterpart. The properties of this source have been investigated in greater detail and are discussed in Sect. 5.2. Finally, in the right hand panel of Fig. 1 we report the case of a correspondence probably due to chance: two close objects, the galaxy IRAS F02374-6130 and the flat spectrum radio quasar PKS 0235-618, are responsible for the hard X-ray and the $\gamma$-ray emission, respectively.

We compare our results with those reported in Abdo et al. (2010b, their Table 7) and find that all the 50 sources 
A. Maselli et al.: The populations of hard X-ray and $\gamma$-ray sources

Table 1. List of correspondences between the 1FGL and 2PBC catalogues at Galactic latitude $|b|>10^{\circ}$.

\begin{tabular}{|c|c|c|c|c|c|c|c|}
\hline 1FGL source & 1FGL counterpart & Type & 2PBC source & 2PBC counterpart & Type & $Q$ & Code \\
\hline \multirow[t]{2}{*}{ 1FGL J0101.3-7257 } & SMC & gal & 2PBC J0102.7-7241 & XTE J0103-728 & HXB & 0.86 & $\mathrm{n}$ \\
\hline & & & & RX J0104.1-7244 & HXB & & \\
\hline 1FGL J0217.8+7353 & 1ES $0212+735$ & bzq & 2PBC J0217.4+7349 & 1ES $0212+735$ & BZQ & 0.47 & $\mathrm{y}$ \\
\hline 1FGL J0238.3-6132 & PKS 0235-618 & bzq & 2PBC J0238.3-6116 & IRAS F02374-6130 & $\mathrm{G}$ & 0.94 & $\mathrm{n}$ \\
\hline 1FGL J0242.7+0007 & RXJ0241.9+0009 (*) & unk & 2PBC J0242.7-0000 & NGC 1068 & Sy2 & 0.88 & $\mathrm{n}$ \\
\hline 1FGL J0319.7+4130 & NGC 1275 & agn & 2PBC J0319.7+4129 & NGC 1275 & $\mathrm{BZU}$ & 0.41 & $\mathrm{y}$ \\
\hline 1FGL J0325.0+3403 & B2 $0321+33 B$ & agn & 2PBC J0324.7+3409 & $1 \mathrm{H} 0323+342$ & Sy1 & 0.46 & $\mathrm{y}$ \\
\hline 1FGL J0334.2+3233 & NRAO $140\left(^{*}\right)$ & bzq & 2PBC J0336.5+3219 & NRAO 140 & BZQ & 1.27: & $\mathrm{y}$ \\
\hline 1FGL J0339.1-1734 & PKS 0336-177 & agn & 2PBC J0339.2-1742 & 1RXS J033913.4-173553 & $X$ & 1.49 & $\mathrm{y}$ \\
\hline 1FGL J0405.6-1309 & PKS 0403-13 & bzq & 2PBC J0405.6-1308 & RX J0405.5-1308 & BZQ & 0.43 & $\mathrm{y}$ \\
\hline 1FGL J0437.2-4715 & PSR J0437-4715 & PSR & 2PBC J0437.8-4713 & RBS 0560 & Sy1 & - & $\mathrm{n}$ \\
\hline 1FGL J0440.6+2748 & B2 0437+27B & bzb & 2PBC J0440.8+2739 & 1RXS J044046.9+273948 & $\mathrm{X}$ & 1.38: & $\mathrm{n}$ \\
\hline 1FGL J0522.8-3632 & PKS 0521-36 & bzb & 2PBC J0523.0-3626 & RBS 0644 & BZU & 1.01 & $\mathrm{y}$ \\
\hline 1FGL J0531.0+1331 & PKS 0528+134 & bzq & 2PBC J0530.9+1333 & PKS $0528+134$ & BZQ & 0.52 & $\mathrm{y}$ \\
\hline 1FGL J0538.8-4404 & PKS 0537-441 & bzb & 2PBC J0538.9-4406 & PKS 0537-441 & $\mathrm{BZB}$ & 0.46 & $\mathrm{y}$ \\
\hline 1FGL J0539.1-2847 & PKS 0537-286 & bzq & 2PBC J0539.8-2839 & PKS 0537-286 & BZQ & 0.56 & $\mathrm{y}$ \\
\hline 1FGL J0557.6-3831 & CRATES J0558-3838 & bzb & 2PBC J0558.0-3821 & Н 0557-385 & Sy1 & 1.21 & $\mathrm{n}$ \\
\hline 1FGL J0636.1-7521 & PKS 0637-75 & bzq & 2PBC J0635.4-7514 & PKS 0637-752 & BZQ & 0.80 & $\mathrm{y}$ \\
\hline 1FGL J0710.6+5911 & BZB J0710+5908 & bzb & 2PBC J0710.2+5909 & $1 \mathrm{H} 0658+595$ & BZB & 0.58 & $\mathrm{y}$ \\
\hline 1FGL J0746.6+2548 & B2 $0743+25$ & bzq & 2PBC J0746.4+2548 & 87GB $074322.5+255639$ & BZQ & 0.31 & $\mathrm{y}$ \\
\hline 1FGL J0750.6+1235 & PKS $0748+126$ & bzq & 2PBC J0750.6+1231 & $\mathrm{OI}+280$ & BZQ & 0.43 & $\mathrm{y}$ \\
\hline 1FGL J0806.2+6148 & CGRaBS J0805+6144 & bzq & 2PBC J0805.4+6146 & GB6 J0805+6144 & BZQ & 0.87 & $\mathrm{y}$ \\
\hline 1FGL J0842.2+7054 & $4 \mathrm{C}+71.07$ & bzq & 2PBC J0841.4+7053 & S5 $0836+71$ & BZQ & 0.37 & $\mathrm{y}$ \\
\hline \multirow[t]{2}{*}{ 1FGL J0929.4+5000 } & CGRaBS J0929+5013 $\left(^{*}\right)$ & bzb & 2PBC J0928.5+4959 & - & - & 0.96 & - \\
\hline & & & 2PBC J0930.6+4954 & RBS 0782 & BZB & 1.29: & $\mathrm{n}$ \\
\hline 1FGL J0949.0+0021 & CGRaBS J0948+0022 & agn & 2PBC J0948.9+0021 & RX J0948.8+0022 & BZQ & 0.27 & $\mathrm{y}$ \\
\hline 1FGL J0956.5+6938 & M 82 & $\operatorname{sbg}$ & 2PBC J0955.7+6941 & M 82 & IG & 0.64 & $\mathrm{y}$ \\
\hline 1FGL J1048.7+8054 & CGRaBS J1044+8054 & bzq & $2 \mathrm{PBC} \mathrm{J} 1044.1+8054$ & S5 1039+81 & BZQ & 1.07 & $\mathrm{y}$ \\
\hline 1FGL J1103.7-2329 & CRATES J1103-2329 & bzb & 2PBC J1103.6-2329 & 1H $1100-230$ & BZB & 0.37 & $\mathrm{y}$ \\
\hline 1FGL J1104.4+3812 & Mkn 421 & bzb & 2PBC J1104.4+3813 & Mrk 421 & BZB & 0.41 & $\mathrm{y}$ \\
\hline 1FGL J1130.2-1447 & PKS 1127-14 & bzq & 2PBC J1130.1-1449 & $\mathrm{OM}-146$ & BZQ & 0.37 & $\mathrm{y}$ \\
\hline 1FGL J1136.2+6739 & BZB J1136+6737 & bzb & 2PBC J1137.2+6735 & RBS 1004 & BZB & 0.18 & $\mathrm{y}$ \\
\hline 1FGL J1221.3+3008 & B2 $1218+30$ & bzb & 2PBC J1221.3+3008 & 1RXS J122121.7+301041 & BZB & 0.12 & $\mathrm{y}$ \\
\hline 1FGL J1222.5+0415 & $4 \mathrm{C}+04.42$ & bzq & 2PBC J1222.3+0415 & $4 \mathrm{C} 04.42$ & BZQ & 0.62 & $\mathrm{y}$ \\
\hline 1FGL J1224.7+2121 & $4 C+21.35$ & bzq & 2PBC J1224.8+2122 & $4 C+21.35$ & BZQ & 0.40 & $\mathrm{y}$ \\
\hline 1FGL J1227.9-4852 & - & - & 2PBC J1228.0-4854 & XSS J12270-4859 & $\mathrm{CV}^{*}$ & 0.22 & - \\
\hline 1FGL J1229.1+0203 & $3 \mathrm{C} 273$ & bzq & 2PBC J1229.1+0202 & $3 \mathrm{C} 273$ & BZQ & 0.25 & $\mathrm{y}$ \\
\hline 1FGL J1256.2-0547 & $3 C 279$ & BZQ & 2PBC J1256.1-0547 & 3C 279 & BZQ & 0.37 & $\mathrm{y}$ \\
\hline 1FGL J1305.4-4928 & NGC 4945 & agn & 2PBC J1305.4-4928 & NGC 4945 & Sy2 & 0.10 & $\mathrm{y}$ \\
\hline 1FGL J1307.0-4030 & ESO $323-77\left({ }^{*}\right)$ & agn & 2PBC J1306.5-4025 & ESO 323-77 & Sy2 & 0.74 & $\mathrm{y}$ \\
\hline 1FGL J1320.1-4007 & - & - & 2PBC J1320.2-4014 & - & - & 0.72 & - \\
\hline 1FGL J1325.6-4300 & Cen A & agn & 2PBC J1325.4-4301 & Cen A & $\mathrm{BZU}$ & 0.61 & $\mathrm{y}$ \\
\hline 1FGL J1331.9-0506 & PKS 1329-049 & bzq & 2PBC J1332.0-0510 & PKS 1329-049 & BZQ & 0.91 & $\mathrm{y}$ \\
\hline 1FGL J1417.8+2541 & $2 \mathrm{E} 1415+2557$ & bzb & 2PBC J1417.9+2543 & 7C $1415+2556$ & $\mathrm{BZB}$ & 0.37 & $\mathrm{y}$ \\
\hline 1FGL J1428.7+4239 & 1ES $1426+428$ & bzb & 2PBC J1428.6+4239 & H $1426+428$ & BZB & 0.58 & $\mathrm{y}$ \\
\hline 1FGL J1442.8+1158 & 1ES $1440+122$ & bzb & 2PBC J1442.8+1202 & RBS 1420 & $\mathrm{BZB}$ & 0.90 & $\mathrm{y}$ \\
\hline 1FGL J1512.8-0906 & PKS 1510-08 & BZQ & 2PBC J1512.8-0906 & PKS 1510-08 & BZQ & 0.42 & $\mathrm{y}$ \\
\hline 1FGL J1517.8-2423 & AP Lib & bzb & 2PBC J1517.7-2419 & Ap Lib & BZB & 0.20 & $\mathrm{y}$ \\
\hline 1FGL J1555.7+1111 & PG $1553+113$ & bzb & 2PBC J1555.5+1109 & PG $1553+113$ & BZB & 0.73 & $\mathrm{y}$ \\
\hline 1FGL J1626.2-2956 & PKS 1622-29 & bzq & 2PBC J1626.0-2952 & PKS 1622-29 & $\mathrm{BZU}$ & 0.85 & $\mathrm{y}$ \\
\hline 1FGL J1642.5+3947 & $3 \mathrm{C} 345\left(^{*}\right)$ & bzq & 2PBC J1643.0+3951 & 4C 39.48 & BZQ & 1.52 & $\mathrm{y}$ \\
\hline 1FGL J1653.9+3945 & Mkn 501 & bzb & 2PBC J1653.8+3945 & Mrk 501 & BZB & 1.16 & $\mathrm{y}$ \\
\hline 1FGL J1829.8+4845 & 3C 380 & agn & 2PBC J1829.6+4845 & 3C 380 & BZU & 0.24 & $\mathrm{y}$ \\
\hline 1FGL J1835.3-3255 & NGC 6652 & glc & 2PBC J1835.7-3259 & XB 1832-330 & LXB & 0.80 & $\mathrm{n}$ \\
\hline 1FGL J1925.2-2919 & PKS B1921-293 & bzq & 2PBC J1924.4-2913 & OV -236 & BZQ & 1.47 & $\mathrm{y}$ \\
\hline
\end{tabular}


Table 1. continued.

\begin{tabular}{lcclcccc}
\hline \hline 1FGL source & 1FGL counterpart & Type & 2PBC source & 2PBC counterpart & Type & $Q$ & Code \\
\hline 1FGL J2000.0+6508 & 1ES 1959+650 & bzb & 2PBC J1959.8+6509 & 1ES 1959+650 & BZB & 1.10 & y \\
1FGL J2011.4-2903 & - & - & 2PBC J2010.8-2910 & - & - & 1.71 & - \\
1FGL J2148.5+0654 & 4C +06.69 & bzq & 2PBC J2148.0+0657 & 4C +06.69 & BZQ & 0.41 & y \\
1FGL J2202.8+4216 & BL Lac & bzb & 2PBC J2202.7+4217 & BL Lac & BZB & 0.24 & y \\
1FGL J2229.7-0832 & PKS 2227-08 & bzq & 2PBC J2229.6-0831 & PKS 2227-08 & BZQ & 0.65 & y \\
1FGL J2232.5+1144 & CTA 102 & bzq & 2PBC J2232.4+1144 & 4C +11.69 & BZQ & 0.11 & y \\
1FGL J2253.9+1608 & 3C 454.3 & BZQ & 2PBC J2253.9+1609 & 3C 454.3 & BZQ & 0.46 & y \\
1FGL J2327.7+0943 & PKS 2325+093 & bzq & 2PBC J2327.4+0939 & PKS J2327+0940 & BZQ & 1.22 & y \\
1FGL J2359.0-3035 & 1H 2351-315 & bzb & 2PBC J2359.1-3035 & H 2356-309 & BZB & 0.28 & y \\
\hline
\end{tabular}

Notes. From Abdo et al. (2010a): gal = normal galaxy; agn = non blazar active galaxy; sbg = starburst galaxy; bzb = BL Lac object; bzq = flat spectrum radio quasar; agu = active galaxy of uncertain type; $\mathrm{psr}=$ pulsar; $\mathrm{pwn}=$ pulsar wind nebula; $\mathrm{spp}=$ potential association with a supernova remnant or a pulsar wind nebula; glc $=$ globular cluster; mqo $=$ microquasar object; $\mathrm{hxb}=$ other X-ray binary. Designations in capital letters correspond to firm identifications. From Abdo et al. (2010b): unk = AGN of unknown type. The nomenclature adopted in Cusumano et al. (2010b) is derived from SIMBAD online services, with the exception of blazars: LXB = low mass X-ray binary; HXB = high mass X-ray binary; $\mathrm{G}=$ normal galaxy; Sy1 = Seyfert 1 galaxy; Sy2 = Seyfert 2 galaxy; $\mathrm{X}=\mathrm{X}$-ray source; $\mathrm{IG}=$ interacting galaxy; $\mathrm{CV}^{*}=$ cataclismic variable star; $\mathrm{PSR}=$ pulsar; $\mathrm{V}^{*}=$ variable star; SNR = superNova remnant; gam = gamma-ray source; $\mathrm{QSO}=$ Quasi Stellar object. The nomenclature of the BZCAT (Massaro et al. 2009) has been used for blazars: BZB = BL Lac objects; BZQ = flat spectrum radio quasars; BZU = blazars with uncertain classification.

Table 2. List of correspondences between the 1FGL and 2PBC catalogues at Galactic latitude $|b|<10^{\circ}$.

\begin{tabular}{|c|c|c|c|c|c|c|c|}
\hline 1FGL source & 1FGL counterpart & Type & 2PBC source & 2PBC counterpart & Type & $Q$ & Code \\
\hline 1FGL J0035.9+5951 & $1 \mathrm{ES} 0033+595$ & bzb & 2PBC J0035.8+5951 & 1ES $0033+59.5$ & BZB & 0.49 & $\mathrm{y}$ \\
\hline 1FGL J0240.5+6113 & LS I+61 303 & HXB & 2PBC J0240.6+6114 & GT 0236+610 & HXB & - & $\mathrm{y}$ \\
\hline 1FGL J0419.0+3811 & 3C 111 & agn & 2PBC J0418.3+3801 & 3C 111 & Sy1 & 0.86 & $\mathrm{y}$ \\
\hline 1FGL J0534.5+2200 & PSR J0534+2200 & PSR & 2PBC J0534.5+2201 & Crab & PSR & - & $\mathrm{y}$ \\
\hline 1FGL J0730.3-1141 & PKS 0727-11 & bzq & 2PBC J0730.4-1142 & PG 0727-11 & BZQ & 0.37 & $\mathrm{y}$ \\
\hline 1FGL J0835.3-4510 & PSR J0835-4510 & PSR & 2PBC J0835.3-4511 & Vela Pulsar & PSR & - & $\mathrm{y}$ \\
\hline 1FGL J1045.2-5942 & - & - & 2PBC J1044.8-5942 & $\mathrm{V}^{*}$ eta $\mathrm{Car}$ & $\mathrm{V}^{*}$ & 0.70 & - \\
\hline 1FGL J1124.6-5916 & PSR J1124-5916 & PSR & 2PBC J1124.9-5919 & SNR G292.0+01.8 & SNR & - & $\mathrm{y}$ \\
\hline 1FGL J1632.7-4733c & - & - & 2PBC J1632.7-4727 & IGR J16328-4726 & gam & 0.87 & - \\
\hline 1FGL J1656.2-3257 & - & - & 2PBC J1656.2-3303 & SWIFT J1656.3-3302 & QSO & 0.85 & - \\
\hline 1FGL J1724.0-3611c & - & - & 2PBC J1725.2-3616 & IGR J17252-3616 & HXB & 0.69: & - \\
\hline 1FGL J1738.5-2656 & - & - & 2PBC J1738.2-2700 & SLX $1735-269$ & LXB & 0.74 & - \\
\hline \multirow[t]{2}{*}{ 1FGL J1746.4-2849c } & PWN G0.13-0.11 & pwn & 2PBC J1746.8-2845 & CXOGCS J174621.05-284343.2 & $\mathrm{X}$ & 1.00 & $\mathrm{n}$ \\
\hline & & & 2PB CJ1746.2-2853 & SAX J1747.0-2853 & LXB & 1.30 & $\mathrm{n}$ \\
\hline 1FGL J1747.2-2958 & PSR J1747-2958 & PSR & 2PBC J1747.4-3000 & 1RXS J174726.8-300008 & LXB & - & $\mathrm{n}$ \\
\hline 1FGL J1747.6-2820c & - & - & 2PBC J1747.6-2820 & CXOGCS J174742.4-282228 & $\mathrm{X}$ & 0.94 & - \\
\hline 1FGL J1826.2-1450 & LS 5039 & HXB & 2PBC J1826.3-1450 & V* V479 Sct & HXB & - & $\mathrm{y}$ \\
\hline 1FGL J1833.5-1034 & PSR J1833-1034 & PSR & 2PBC J1833.5-1033 & SNR 021.5-00.9 & PSR & - & $\mathrm{y}$ \\
\hline 1FGL J1833.6-2103 & PKS 1830-21 & bzq & 2PBC J1833.7-2102 & PKS 1830-211 & BZQ & 0.34 & $\mathrm{y}$ \\
\hline 1FGL J2015.7+3708 & - & - & 2PBC J2015.9+3712 & RX J2015.6+3711 & $\mathrm{CV}^{*}$ & 0.95 & - \\
\hline 1FGL J2032.4+4057 & Cyg X-3 & MQO & 2PBC J2032.4+4057 & Cyg X-3 & HXB & - & $\mathrm{y}$ \\
\hline 1FGL J2056.7+4938 & - & - & 2PBC J2056.5+4938 & RX J2056.6+4940 & $\mathrm{X}$ & 0.43 & - \\
\hline 1FGL J2323.4+5849 & - & spp & 2PBC J2323.3+5849 & Cas A & SNR & 0.21 & - \\
\hline
\end{tabular}

Notes. See notes of Table 1.

provided in their list are included in Table 1, with the exception of 1FGL J1938.2-3957. Hard X-ray emission in this direction was revealed by INTEGRAL and reported in the fourth IBIS catalogue (Bird et al. 2010). The counterpart associated to this 1FGL source, PKS 1933-400, is also reported in the BZCAT as a blazar with uncertain classification. The value $\sigma \sim 3.5$ that we find in the BAT $15-150 \mathrm{keV}$ all-sky map at the position of PKS 1933-400 is compatible with hard X-ray emission from this source, but it is lower than $\sigma_{\mathrm{T}}=4.8$.

\section{Correspondences of 1FGL sources with the 54-month hard X-ray maps}

The 2PBC catalogue includes sources detected at a significance level higher than $\sigma_{\mathrm{T}}=4$.8. A remarkable number of fainter objects are presumably imaged in the 54-month BAT all-sky maps at a lower significance level. As an effort towards increasing the list of objects emitting both in the BAT and in the LAT energy ranges, we consider the adoption of a lower threshold of the detection significance. Taking into account the results obtained by Maselli et al. (2010) in the cross-correlation of the BZCAT Blazar Catalogue (Massaro et al. 2009) with the 39-month 15-150 keV BAT map (Cusumano et al. 2010a) we adopt $\sigma_{\mathrm{T}}^{\star}=3$. From their analysis, carried out at $|b|>10^{\circ}$, Maselli et al. (2010) find that the adoption of $\sigma_{\mathrm{T}}^{\star}$ follows in a fraction of $\sim 3 \%$ of related spurious associations, but this value is presumably underestimated at $|b|<10^{\circ}$ owing to the higher density of sources.

A total of 1043 and 408 objects are found in the 1FGL catalogue at high $\left(|b|>10^{\circ}\right)$ and low Galactic latitude, respectively. 
A. Maselli et al.: The populations of hard X-ray and $\gamma$-ray sources

Table 3. List of 1FGL sources at Galactic latitude $|b|>10^{\circ}$ considering a significance threshold $\sigma_{\mathrm{T}}^{\star}=3$ in the BAT $15-150 \mathrm{keV}$ all-sky map.

\begin{tabular}{lccl}
\hline \hline 1FGL source & 1FGL counterpart & Type & BZCAT source \\
\hline 1FGL J0137.5-2428 & PKS 0135-247 & bzq & BZQ J0137-2430 \\
1FGL J0144.9-2732 & PKS 0142-278 & bzq & BZQ J0145-2733 \\
1FGL J0334.2-4010 & PKS 0332-403 & bzb & BZB J0334-4008 \\
1FGL J0403.9-3603 & PKS 0402-362 & bzq & BZQ J0403-3605 \\
1FGL J0455.6-4618 & PKS 0454-46 & bzq & BZQ J0455-4615 \\
1FGL J0507.3-6103 & CRATES J0507-6104 & bzq & BZQ J0507-6104 \\
1FGL J0507.9+6738 & 1ES 0502+675 & bzb & BZB J0507+6737 \\
1FGL J0639.9+7325 & CGRaBS J0639+7324 & bzq & BZQ J0639+7324 \\
1FGL J0721.9+7120 & S5 0716+714 & bzb & BZB J0721+7120 \\
1FGL J0739.1+0138 & PKS 0736+01 & bzq & BZQ J0739+0137 \\
1FGL J1031.0+5051 & 1ES 1028+511 & bzb & BZB J1031+5053 \\
1FGL J1159.4+2914 & 4C +29.45 & bzq & BZQ J1159+2914 \\
1FGL J1354.9-1041 & PKS 1352-104 & bzq & BZU J1354-1041 \\
1FGL J1604.3+5710 & CGRaBS J1604+5714 & bzq & BZQ J1604+5714 \\
1FGL J1617.9-7716 & PKS 1610-77 & bzq & BZQ J1617-7717 \\
1FGL J1637.9+4707 & 4C +47.44 & bzq & BZQ J1637+4717 \\
1FGL J1725.0+1151 & CGRaBS J1725+1152 & bzb & BZB J1725+1152 \\
1FGL J1800.4+7827 & CGRaBS J1800+7828 & bzb & BZB J1800+7828 \\
1FGL J1849.3+6705 & CGRaBS J1849+6705 & bzq & BZQ J1849+6705 \\
1FGL J1923.5-2104 & OV -235 & bzq & BZQ J1923-2104 \\
1FGL J2143.4+1742 & OX 169 & bzq & BZQ J2143+1743 \\
1FGL J2158.8-3013 & PKS 2155-304 & bzb & BZB J2158-3013 \\
\hline
\end{tabular}

Notes. See notes of Table 1.

Table 4. List of 1FGL sources at Galactic latitude $|b|<10^{\circ}$ considering a significance threshold $\sigma_{\mathrm{T}}^{\star}=3$ in the BAT $15-150 \mathrm{keV}$ all-sky map.

\begin{tabular}{lccc}
\hline \hline 1FGL source & 1FGL counterpart & Type & BZCAT source \\
\hline 1FGL J0137.8+5814 & - & - & - \\
1FGL J0849.6-3540 & VCS2 J0849-3541 & agu & - \\
1FGL J1329.2-5605 & PMN J1329-5608 & agu & - \\
1FGL J1420.1-6048 & PSR J1420-6048 & PSR & - \\
1FGL J1714.5-3830c & - & - & - \\
1FGL J2021.0+3651 & PSR J2021+3651 & PSR & - \\
1FGL J2347.1+5142 & 1ES 2344+514 & bzb & BZB J2347+5142 \\
\hline
\end{tabular}

Notes. See notes of Table 1 .

We extracted the value of the detection significance in the 54month 15-150 keV BAT all-sky map at their positions and find $\sigma \geq \sigma_{\mathrm{T}}^{\star}$ for 80 objects at $|b|>10^{\circ}$ and 49 objects at $|b|<10^{\circ}$. In a few of these objects, the $\sigma$ value may be strongly biased by some close, very bright hard X-ray sources. Following the same criterion as adopted in Maselli et al. (2010), we excluded the 1FGL sources whose position is found within $36^{\prime}$ from these bright BAT sources and no overlap, even marginal, is found between the corresponding error regions. After this screening we obtain a sample of $75\left(|b|>10^{\circ}\right)$ and $29\left(|b|<10^{\circ}\right)$ 1FGL sources. All the sources listed in Tables 1 and 2 are included in this sample with nine exceptions, all of them at high Galactic latitude. They concern 1FGL sources for which the $95 \%$ confidence ellipse is particularly wide and where the $2 \mathrm{PBC}$ source is at a considerable distance from the centre of the ellipse. The final list of additional sources with respect to those obtained from the cross-correlation between the 1FGL and 2PBC catalogues has been split according to the Galactic latitude and reported in Tables 3 and 4. For each source the association and the classification type indicated in the 1FGL catalogue is reported, together with the BZCAT blazar classification.

\section{Properties of the resulting samples of sources}

The firm correspondences that we find by cross-correlating the 1FGL and $2 \mathrm{PBC}$ catalogues and verifying the agreement between the identified counterparts lead to 62 sources. This number rises to 104 ( $\sim 7 \%$ of all the 1FGL objects) when considering all the sources detected with a significance down to $\sigma_{\mathrm{T}}^{\star}=3$, therefore the number of sources emitting in both the energy ranges covered by the BAT and the LAT instruments aboard Swift and Fermi, respectively, is small. This is a clear indication that the emission in the hard X-ray and in the $\gamma$-ray sky is dominated by sources of a different nature. This result is expected, because among extragalactic sources the main contribution in the 1FGL catalogue is given by blazars, while in the $2 \mathrm{PBC}$ catalogue it is given by Seyfert galaxies. Considering the 1FGL extragalactic sources provided with an association, the blazar contribution is given by 295 BL Lacs and 274 FSRQs: active galaxies with uncertain classification are 92, while nonblazar active galaxies are only 28 . Conversely, in the $2 \mathrm{PBC}$ catalogue there are 307 Seyfert 1 and 165 Seyfert 2 galaxies. Even if considerable, the blazar contribution ( 97 objects) is less relevant.

The group of 104 objects that we have collected is made up of 82 extragalactic and 15 Galactic sources, while seven objects are unidentified. The largest number of extragalactic sources are given by blazars with only a very few exceptions: the Seyfert 1.2 galaxy ESO 323-77, the FR II radiogalaxy 3C 111, the flat spectrum radio source PKS 0336-177 and the starburst galaxies M 82 and NGC 4945. At a low Galactic latitude, we find six 

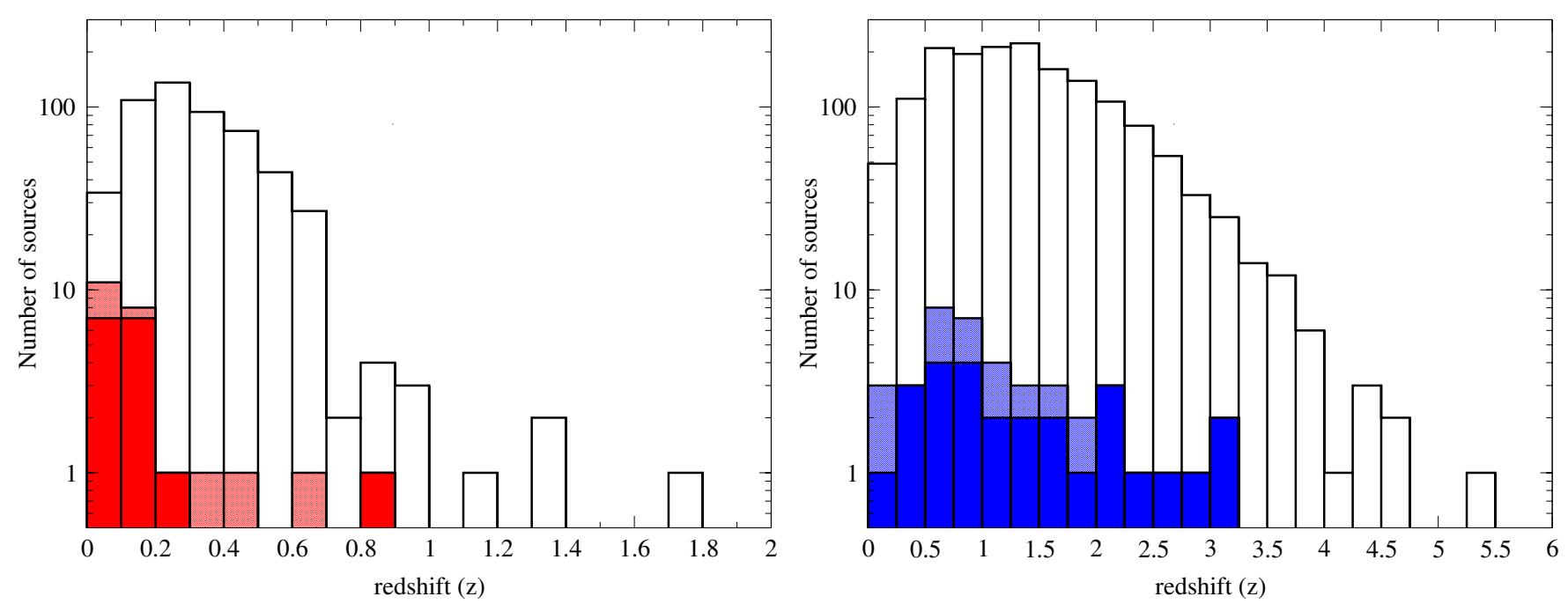

Fig. 2. Histograms of the redshift distributions for BL Lac objects (left panel) and flat spectrum radio quasars (right panel). The contribution of 1FGL sources detected with a significance down to $\sigma_{\mathrm{T}}^{\star}=3$ in the BAT $15-150 \mathrm{keV}$ all-sky map (filled columns) is compared with that of the whole corresponding population reported in the BZCAT (empty columns). The contribution of sources included in the $2 \mathrm{PBC}$ catalogue is emphasised by an unspotted colour.

Table 5. The mean redshift values of different blazar subsamples showing emission in both the BAT and the LAT energy ranges, compared to those of the corresponding blazar population as reported in the BZCAT.

\begin{tabular}{lcccccc}
\hline \hline Blazar type & 1FGL-2PBC & $\left\langle z_{1}\right\rangle$ & $\sigma>\sigma_{\mathrm{T}}^{\star}$ & $\left\langle z_{2}\right\rangle$ & BZCAT & $\langle z\rangle$ \\
\hline BZB & $16(14)$ & $0.17 \pm 0.06$ & $24(19)$ & $0.21 \pm 0.05$ & $1164(531)$ & $0.33 \pm 0.01$ \\
BZQ & 27 & $1.39 \pm 0.17$ & 41 & $1.23 \pm 0.12$ & $1660(1638)$ & $1.40 \pm 0.02$ \\
BZU & 7 & $0.32 \pm 0.14$ & 8 & $0.32 \pm 0.12$ & $262(215)$ & $0.42 \pm 0.04$ \\
\hline total & $50(48)$ & & $73(68)$ & & $3086(2384)$ & \\
\hline
\end{tabular}

pulsars (including Crab and Vela), four high-mass X-ray binaries (including Cyg X-3), a low-mass X-ray binary, the supernova remnant Cas A, two cataclysmic variable stars, and the peculiar object Eta Carinae. We briefly report the results obtained after considering the even lower value of the significance threshold $\sigma_{\mathrm{T}}=2$. We find 74 more sources, the largest fraction of which are blazars (16 BZB, $18 \mathrm{BZQ}$, and $9 \mathrm{BZU})$, followed by other AGNs (among which is the well known FR I radiogalaxy M87), the starburst galaxy NGC 253, and a few pulsars; 14 sources are unidentified. We note that, according to Maselli et al. (2010), the number of spurious correspondences for such a low $\sigma_{\mathrm{T}}$ value is $\sim 20 \%$.

We focused on the blazar content of the 1FGL-2PBC sources and investigate their distance. We followed the classification of the second edition of the BZCAT catalogue (Massaro et al. 2010), which includes 1164 BL Lac objects (indicated in the following with the suffix "B"), 1660 flat spectrum radio quasars (suffix "Q"), and 262 blazars with uncertain classification (suffix "U"). Note that a $z$ value is available for 531 BL Lacs and therefore the others were discarded in this analysis. We computed the mean redshift value of the different classes of 1FGL-2PBC blazars: the results are reported in Table 5 . The values $\left\langle z_{1}\right\rangle$ refer to the groups of blazars obtained from the cross-correlation of the 1FGL and $2 \mathrm{PBC}$ catalogues, while $\left\langle z_{2}\right\rangle$ refer to the larger groups obtained by adopting $\sigma_{T}^{\star}=3$ as a threshold for the detection significance in the BAT $15-150 \mathrm{keV}$ all-sky map. Values in brackets refer to sources characterised by redshift estimates that are firmly established and different from zero. We note that five among the $24 \mathrm{BL}$ Lacs detected above $\sigma_{\mathrm{T}}^{\star}$ do not have any redshift estimate, two of which are included in the $2 \mathrm{PBC}$ catalogue. We compared these results with the values $\langle z\rangle$ that characterise the blazar subclasses as a whole, computed by considering the totality of sources classified in the BZCAT.

We plot in Fig. 2 the histograms of the redshift distributions for BL Lacs (left panel) and flat spectrum radio quasars (right panel) for these groups of sources. Our results show that the subsample of blazars emitting both in the BAT and in the LAT energy bands is made up of sources that are relatively closer than average to the observer. In fact, $\left\langle z_{2}\right\rangle$ is lower than the mean redshift value $\langle z\rangle$ computed for all the different blazar subclasses, as reported in Table 5 . The analysis of the plot in the left hand panel of Fig. 2 shows that the largest part of BL Lac objects coming from the cross-correlation of 1FGL and 2PBC catalogues has a redshift $z_{\mathrm{B}}<0.2$, while the modal value of the BL Lac distribution is in the range $0.2<z_{\mathrm{B}}<0.3$. Adding sources with a detection significance down to $\sigma_{\mathrm{T}}^{\star}=3$ confirms this result, where most have $z_{\mathrm{B}}<0.1$, with the remaining sources more or less equally distributed at higher redshift up to $z_{\mathrm{B}}=0.7$. An analogous result is found for flat-spectrum radio quasars (Fig. 2, right panel): high-energy sources emitting both in the BAT and in the LAT energy bands have redshift $z_{Q}<3.2$, with a peak in the range $0.5<z_{Q}<1$. The addition of sources with a detection significance down to $\sigma_{\mathrm{T}}^{\star}=3$ adds seven more sources to this peak, and none with a redshift higher than $z_{Q}=2$. Conversely, the modal value of the distribution of all the FSRQs catalogued in the BZCAT is higher than this peak, in the range $1.25<z_{Q}<1.5$. 


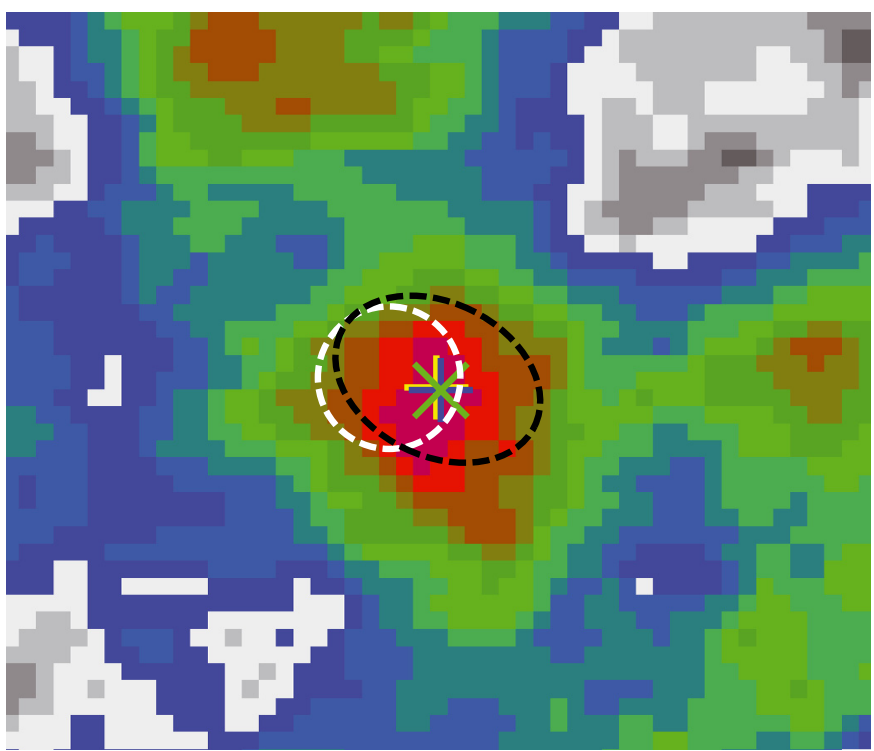

Fig. 3. The BAT $15-150 \mathrm{keV}$ all-sky map $\left(60^{\prime} \times 60^{\prime}\right)$ of the detection significance in the field of 1FGL J0137.8+5814. The positions of the Fermi-LAT and the INTEGRAL-IBIS detections with the corresponding uncertainty regions are plotted with black and a white dashed lines, respectively. The positions of the soft X-ray detections (ROSAT: yellow cross; XMM-Newton: green cross; Swift-XRT: blue cross) are very close to each other and practically coincident to that of the optical counterpart.

\section{New possible associations of sources in the BAT and LAT surveys}

The high-energy emission revealed by the BAT and the LAT telescopes is very helpful in classifying already known sources more correctly. Moreover, it can lead to the discovery of new blazars in regions where their identification is complicated by the belt surrounding the Galactic plane intercepting the line of sight towards them. We focused our attention on two 1FGL unidentified sources, 1FGL J0137.8+5814 and 1FGL J2056.7+4938, detected at low Galactic latitude and included in Tables 2 and 4. For each source we built the SED by adding the data obtained from our reduction of Swift and XMMNewton pointed observations to all the data that we found in the literature.

Swift-XRT observations, carried out using the most sensitive photon-counting readout mode (see Hill et al. 2004, for a description of readout modes), are available for both 1FGL J0137.8+5814 and 1FGL J2056.7+4938. The highest detected count rate for each source is, in all cases, lower than the pile-up threshold $\left(0.5 \mathrm{cts} \mathrm{s}^{-1}\right)$. We reduced the data with the HEASOFT 6.8 package distributed by the NASA High Energy Astrophysics Archive Research Center (HEASARC). All the files necessary for the spectral analysis were obtained using the XRTPIPELINE and the XRTPRODUCTS tasks. For each source two Swift observations, characterised by very similar values for the count rate, are available; therefore, we decided to sum the two event files using the XSELECT task and the exposure maps to correct for vignetting and for CCD hot and damaged pixels. We used the XRTCENTROID task to detect the centroid of the source and the corresponding positional error. A nearby source-free region was chosen for extracting the background spectrum. Spectral data of sources were extracted in circular regions surrounding the centroid, adopting a radius of 20 pixels $\left(1\right.$ pixel $\left.=2.36^{\prime \prime}\right)$ for the source spectrum and of 50 pixels for the background spectrum.
A pointed observation from XMM-Newton is available for 1FGL J0137.8+5814. We restricted our analyses of this observation to MOS1 and MOS2, discarding PN data because the source is located at the edge of the instrument's FoV. We used the standard analysis software SAS 10.0 to extract high-level science products from the ODF files. The source spectrum is extracted by selecting all the events with PATTERN $\leq 12$ (restricting the patterns to single and doubles) and FLAG $=0$ within a circle of $40^{\prime \prime}$ radius centred on the source. Similarly, the background was extracted by collecting all the counts within an annulus, centred on the source, with inner and outer radii of $45^{\prime \prime}$ and $85^{\prime \prime}$, respectively. We combined the instrumental channels in the spectral files to include at least 20 counts in each new energy bin for the Swift observation and 25 counts in the case of XMM-Newton. The spectral analysis was carried out using XSPEC 12.5.1n.

The flux density of both sources in the hard X-ray domain $(20 \mathrm{keV})$ was obtained converting their count rate in the 15-30 keV map of the 54-month BAT survey. The conversion factor was calculated from the count rate of Crab and its spectrum used for calibration purposes, as explained in the BAT calibration status report ${ }^{1}$.

The measurements available for these objects at different frequencies are mostly non-simultaneous, and there are not enough of them to allow the possibility of analysing their intrinsic variability at different epochs in detail. We evaluated their general emission properties by selecting opportune energy ranges and, whenever possible, by fitting the corresponding data with analytical models to estimate some relevant parameters. The data in the radio band are well fitted by a power-law model $S(v) \propto v^{-\alpha_{r}}$ to derive their spectral index $\alpha_{r}$. We adopted a logparabolic model to fit the curvature of both synchrotron and inverse Compton components that characterise the SED of blazars. This model, expressed by the analytical formula

$v F(v)=v_{\mathrm{p}} F\left(v_{\mathrm{p}}\right) \times 10^{-\beta\left(\log v / v_{\mathrm{p}}\right)^{2}}$,

provides the peak frequency $v_{\mathrm{p}}$ of the component and the parameter $\beta$ that describes its curvature at the peak. This law reproduces curved spectra with a small number of parameters and has been verified to fit the broad band spectra of blazars well (Landau et al. 1986; see also Massaro et al. 2004a,b, for an interpretation in terms of statistical acceleration).

We note that the Galactic latitude of these sources is very low. For this reason the flux density in the optical band may be severely affected from uncertainties of the Galactic extinction values, which are not supposed to be fully reliable. As regards the $\gamma$-ray data, derived from the 1FGL catalogue, we consider the possibility of contamination due not only to the $\gamma$-ray background but also to the occasional presence of neighbour sources in the field as for the case of 1FGL J0137.8+5814, with a pulsar at an angular distance of $\sim 12^{\prime}$.

\subsection{FGL J0137.8+5814}

We assume from the 1FGL catalogue the position of 1FGL J0137.8+5814 $\left(\mathrm{RA}=01^{\mathrm{h}} 37^{\mathrm{m}} 48^{\mathrm{s}} .77\right.$; Dec $\left.=+58^{\circ} 14^{\prime} 57^{\prime \prime} .1\right)$ with an error radius 7'.5, and the Galactic latitude is $b=-4^{\circ} .07$. The analysis of the 54-month BAT all-sky map of the detection significance shows a considerable hard X-ray emission at this position, but no source has been included in the $2 \mathrm{PBC}$ catalogue (Cusumano et al. 2010b) because its detection significance $(3.4 \sigma)$ is below the threshold $\sigma_{\mathrm{T}}=4.8$ established in this

\footnotetext{
${ }^{1}$ http://swift.gsfc.nasa.gov/docs/swift/analysis/bat_ digest.html
} 
catalogue. In this case, determining the position of the source is a delicate task, so we report the coordinates of the pixel with the locally higher value of the detection significance $(3.5 \sigma)$ : $\mathrm{RA}=01^{\mathrm{h}} 37^{\mathrm{m}} 48.77$ and Dec $=+58^{\circ} 11^{\prime} 21^{\prime \prime}{ }^{\mathrm{s}} 1$. A hard X-ray detection in this region of the sky by INTEGRAL was first included in the all-sky survey by Krivonos et al. (2007). It is also reported in the 4th IBIS/ISGRI catalogue (Bird et al. 2010) with $\mathrm{RA}=01^{\mathrm{h}} 37^{\mathrm{m}} 22^{\mathrm{s}} .8$, Dec $=+58^{\circ} 15^{\prime} 03^{\prime \prime} .6$ and with an error radius of $5^{\prime}$, thus shrinking the region needed to search for a counterpart to the Fermi $\gamma$-ray source. The variability of this object in the $20-40 \mathrm{keV}$ band has been recently assessed by Telezhinsky et al. (2010), where it is still classified as "unidentified" and as a transient source detected on the intrinsic variance map but not on the significance map.

At lower energies, a source in the LAT and IBIS error boxes has been reported in the 1RXS catalogue (Voges et al. 1999) at $\mathrm{RA}=01^{\mathrm{h}} 37^{\mathrm{m}} 48^{\mathrm{s}} .0$, Dec $=+58^{\circ} 14^{\prime} 22^{\prime \prime} .5$ with an error radius of $9^{\prime \prime}$. Later on, an X-ray observation performed by XMMNewton on January 16, 2003 and aimed at observing the pulsar PSR 0136+57 revealed a serendipitous source that was included in the 2XMMi catalogue (Watson et al. 2009) with coordinates $\mathrm{RA}=01^{\mathrm{h}} 37^{\mathrm{m}} 50^{\varsigma} \cdot 4$, Dec $=+58^{\circ} 14^{\prime} 10^{\prime \prime}$ and an error radius of $1^{\prime \prime}$. We analysed this XMM-Newton observation, characterised by a long exposure time $t_{\exp }=8439 \mathrm{~s}$, and considered the results obtained by the combined analysis of MOS1 and MOS2 detectors. We fit the spectrum with a power-law model and obtain a photon index $\Gamma=(2.31 \pm 0.06)$. The value of the hydrogen column density $N_{\mathrm{H}}=(4.96 \pm 0.18) \times 10^{21} \mathrm{~cm}^{-2}$ that we obtain when leaving this parameter free to vary is very similar to the Galactic one $\left(N_{\mathrm{H}}=4.01 \times 10^{21} \mathrm{~cm}^{-2}\right)$ as reported in the Leiden/Argentine/Bonn (LAB) Survey (Kalberla et al. 2005). The source flux is $1.04 \times 10^{-11} \mathrm{erg} \mathrm{cm}^{-2} \mathrm{~s}^{-1}$ in the $2-10 \mathrm{keV}$ band and is $1.5 \times 10^{-11} \mathrm{erg} \mathrm{cm}^{-2} \mathrm{~s}^{-1}$ in the $0.2-12 \mathrm{keV}$ band. We note the lower value of the flux $1.8 \times 10^{-12} \mathrm{erg} \mathrm{cm}^{-2} \mathrm{~s}^{-1}$ in the 2-10 keV band reported by Stephen et al. (2010) and attribute it to the different value of the photon index $\Gamma=1.7$ that they adopted in their fit.

Two Swift observations have been recently obtained in this region: the XRT exposure of the first observation (2010 September 4) is $1153 \mathrm{~s}$, while a longer exposure of $3393 \mathrm{~s}$ is available for the second observation (2010 October 22). Across this period the source has not shown appreciable variations in activity, with a stable count rate around $\sim 1.7 \times 10^{-1} \mathrm{cts} \mathrm{s}^{-1}$. The position of the XRT source is RA $=01^{\mathrm{h}} 37^{\mathrm{m}} 50^{\mathrm{s}} .37$ and Dec $=$ $+58^{\circ} 14^{\prime} 11^{\prime \prime}$. 5 with an error radius $3.61^{\prime}$. We fit the obtained spectrum with a power-law model and find $\chi_{r}^{2} /$ d.o.f. $=0.96 / 35$. As for the XMM-Newton observation, the obtained value for the hydrogen column density $N_{\mathrm{H}}=(4.75 \pm 0.49) \times 10^{21} \mathrm{~cm}^{-2}$ can be considered consistent with the Galactic one. The value of the photon spectral index is $\Gamma=(2.21 \pm 0.14)$, while the obtained value for the $2-10 \mathrm{keV}$ flux is $5.67 \times 10^{-12} \mathrm{erg} \mathrm{cm}^{-2} \mathrm{~s}^{-1}$, which is nearly half the value measured by XMM-Newton in 2003.

Possibly interesting radio counterparts are in the $87 \mathrm{~GB}$ and NVSS (Condon et al. 1998) catalogues (RA $=01^{\mathrm{h}} 37^{\mathrm{m}} 50$ s 46 ; Dec $=+58^{\circ} 14^{\prime} 11^{\prime \prime} .2$ ) with flux densities of $F_{5 \mathrm{GHz}}=136 \mathrm{mJy}$ and $F_{1.4 \mathrm{GHz}}=170 \mathrm{mJy}$, respectively, from which we derive a spectral index $\alpha_{r} \simeq 0.28$. The image in the NVSS survey shows a marginally extended source with a very compact core.

Optical observations (Bikmaev et al. 2008) aimed at identifying the five INTEGRAL sources reported by Krivonos et al. (2007), performed with the Russian-Turkish $1.5-\mathrm{m}$ RTT-150 and the 6-m BTA telescopes, led to the discovery of an object having a continuum without emission or absorption lines, located at RA $=01^{\mathrm{h}} 37^{\mathrm{m}} 50^{\Im} .45$ and Dec $=+58^{\circ} 14^{\prime} 11^{\prime \prime} .6$,

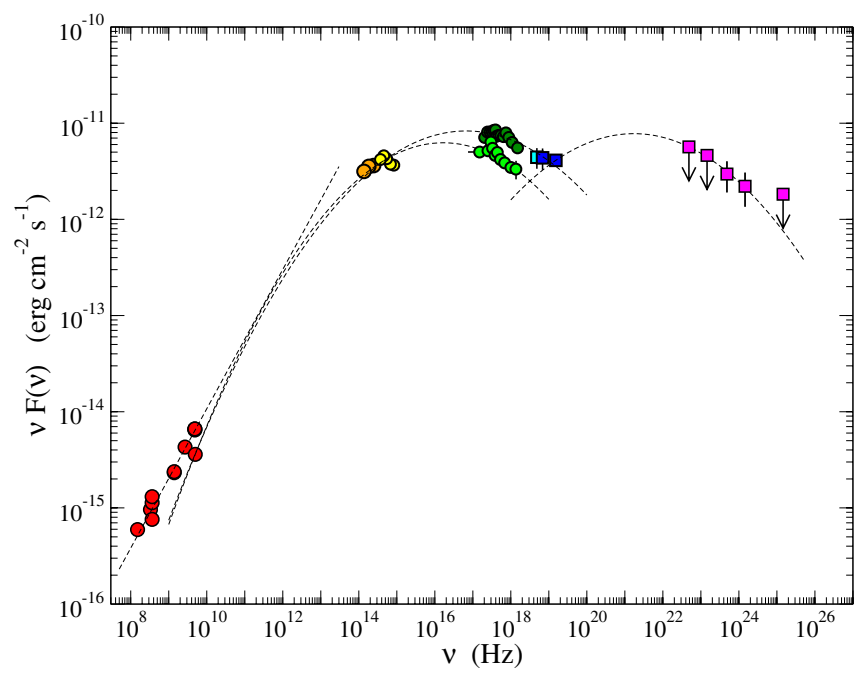

Fig. 4. The SED of 1FGL J0137.8+5814. From lower to higher frequencies we report radio data up to $5 \mathrm{GHz}$ (red circles) and data from 2MASS (orange circles), SDSS-DR8 (yellow circles), SwiftXRT (light green circles), XMM-MOS (dark green circles), Swift-BAT (cyan square), Integral-IBIS (blue squares), and Fermi-LAT (magenta squares). Data are fitted with a power-law model in the radio band; a log-parabolic model is used to emphasise the synchrotron and inverse Compton component of this high-energy synchrotron peak (HSP) BL Lac object.

fully compatible with the radio and X-ray position and at a separation of $\sim 0.8^{\prime}$ from the $\gamma$-ray centroid. Photometric data of the object in the field are available from the Sloan Digital Sky Survey (SDSS-DR8). The morphological classification is that of a starlike source and the magnitudes are $r=(18.12 \pm 0.01) \mathrm{mag}$, $g=(19.05 \pm 0.01) \mathrm{mag}$, and $u=(20.04 \pm 0.05) \mathrm{mag}$, with a reddening in the $r$ band equal to $1.47 \mathrm{mag}$. This implies a correction to the $u-r$ colour index of $\sim 1.2 \mathrm{mag}$, and the intrinsic colour index would be $\sim 0.7 \mathrm{mag}$, corresponding to a spectral distribution with a strong excess at blue wavelengths, typical of BL Lac objects.

Considering all these available data, we obtain the SED of this source shown in Fig. 4. It looks very similar to an SED for an LAT blazar (Abdo et al. 2010c) with the two broad bumps associated with the synchrotron and inverse Compton emission. We estimate the peak frequency $v_{\mathrm{p}}$ and the curvature parameter $\beta$ of both components by fitting a log-parabolic law to the data. The observations of the X-ray telescopes, which monitor the region close to the synchrotron peak in two different epochs, have shown relevant variability in emission from this source. The results of the log-parabolic fit that includes Swift data are $v_{\mathrm{S}}=1.66 \times 10^{+16} \mathrm{~Hz}$ for the peak frequency with a corresponding maximum of synchrotron emission $v_{\mathrm{S}} F\left(v_{\mathrm{S}}\right)=6.25 \times$ $10^{-12} \mathrm{erg} \mathrm{cm}^{-2} \mathrm{~s}^{-1}$, and the estimated value of the curvature parameter is $\beta_{\mathrm{S} 1}=0.08$. The log-parabolic fit that includes $X M M$ Newton data provides a higher value $\left(v_{\mathrm{S}}=6.69 \times 10^{+16} \mathrm{~Hz}\right)$ for the synchrotron peak frequency and a corresponding maximum of synchrotron emission $v_{\mathrm{S}} F\left(v_{\mathrm{S}}\right)=8.30 \times 10^{-12} \mathrm{erg} \mathrm{cm}^{-2} \mathrm{~s}^{-1}$. A very similar curvature $\left(\beta_{\mathrm{S} 2}=0.07\right)$ was also found in this case. As regards the inverse Compton component, we obtain $v_{\mathrm{IC}}=1.78 \times 10^{+21} \mathrm{~Hz}$ with a corresponding $v_{\mathrm{IC}} F\left(v_{\mathrm{IC}}\right)=7.78 \times$ $10^{-12} \mathrm{erg} \mathrm{cm}^{-2} \mathrm{~s}^{-1}$, and the curvature parameter is $\beta_{\mathrm{IC}}=0.07$. Both the flux at the peak frequency and the curvature parameter that we derive from our log-parabolic models are therefore 
very similar for the synchrotron and the inverse Compton components.

A BL Lac classification for this source, which was first suggested by Bikmaev et al. (2008), has been recently confirmed by Stephen et al. (2010). Following the classification scheme reported in Abdo et al. (2010c) we can conclude from our analysis that this object is a high-energy synchrotron peak (HSP) BL Lac object with a $v_{\mathrm{S}}>10^{+16} \mathrm{~Hz}$ in different detected states of the source's activity.

\section{2. $1 F G L ~ J 2056.7+4938$}

The position of 1 FGL J2056.7+4938 $\left(\mathrm{RA}=20^{\mathrm{h}} 56^{\mathrm{m}} 43.51\right.$, Dec $=+49^{\circ} 38^{\prime} 37^{\prime \prime} .3$, corresponding to the Galactic latitude $b=2.74$ ), can be assumed with an error radius $r=$ 2.7 from the 1FGL catalogue. In the $2 \mathrm{PBC}$ there is the source $2 \mathrm{PBC} \mathrm{J} 2056.5+4938$ close to this location at RA = $20^{\mathrm{h}} 56^{\mathrm{m}} 32^{\mathrm{s}} .66$, Dec $=+49^{\circ} 38^{\prime} 30^{\prime} \cdot 3$ with an error radius $\sim 3.5$ at the $95 \%$ confidence level. The significance of the detection is $5.5 \sigma$ in the $15-150 \mathrm{keV}$ map and $6.3 \sigma$ in the $15-30 \mathrm{keV}$ map. Earlier than Swift-BAT, a detection in the hard X-ray band in the proximity of 1FGL J2056.7+4938 was obtained by INTEGRAL-IBIS. The source, first reported by Krivonos et al. (2007) with the name IGR J20569-4940, was left without classification, and the detection was later confirmed by Bird et al. (2010) in their 4th IBIS/ISGRI soft gamma-ray survey catalogue.

The counterpart of this high-energy emission can be searched by shrinking the region of the sky with circles centred on the positions of Fermi-LAT, Swift-BAT and INTEGRAL-IBIS centroids with a radius proportional to their errors, respectively. The radio source $4 \mathrm{C}+49.35$, classified as symmetric double in NED, is found within the intersection of these circles. Radio measurements at $1.4 \mathrm{GHz}$ reported in the NVSS catalogue (Condon et al. 1998) resolve two components separated by $\sim 3^{\prime}$ : a north-east component $\left(\mathrm{RA}=20^{\mathrm{h}} 56^{\mathrm{m}} 42^{\mathrm{s}} .69\right.$, Dec $=+49^{\circ} 40^{\prime} 05^{\prime \prime}$. 6 ) with a flux density $F_{\mathrm{NE}}=167 \mathrm{mJy}$ and a south-west component $\left(\mathrm{RA}=20^{\mathrm{h}} 56^{\mathrm{m}} 29.45\right.$, Dec $=$ $+49^{\circ} 38^{\prime} 01^{\prime \prime}$. 0 ) with $F_{\text {SW }}=124$ mJy.

A soft X-ray emission was detected in this region for the first time by the Ariel V satellite and reported by Warwick et al. (1981) with the name 3A 2056+493. Later detections by ROSAT and, more recently, by Swift and XMM-Newton are characterised by adequate precision to associate the soft X-ray emission with the north-east component. Two detections from XMM-Newton have been reported in the slew survey clean source catalogue (Saxton et al. 2008). Their positions are very similar and separated by $\sim 2.5^{\prime \prime}$ from each other: RA = $20^{\mathrm{h}} 56^{\mathrm{m}} 42^{\mathrm{s}} .84$, Dec $=+49^{\circ} 40^{\prime} 03^{\prime \prime} \cdot 8$ for the first one and RA $=$ $20^{\mathrm{h}} 56^{\mathrm{m}} 42.59$; Dec $=+49^{\circ} 40^{\prime} 04^{\prime \prime} \cdot 3$ for the second one. The error on the position is $8^{\prime \prime}$ at the $1 \sigma$ confidence. Even though the two slews were carried out along the same day (November 03, 2007) flux variations in the $0.2-12 \mathrm{keV}$ band have been reported in the catalogue, dropping from $(1.85 \pm 0.31)$ to $(0.83 \pm 0.25) \times$ $10^{-11} \mathrm{erg} \mathrm{cm}^{-2} \mathrm{~s}^{-1}$ in a few hours.

Two soft X-ray pointed observations were performed by Swift on 2006 February 26 and 2009 March 03. The first one has a much longer exposure (8377 s) than the other (1439 s). We stack the two observations and obtain RA $=20^{\mathrm{h}} 56^{\mathrm{m}} 42^{\mathrm{s}} .68$; $\mathrm{Dec}=+49^{\circ} 40^{\prime} 07^{\prime \prime} .69$ for the position of the X-ray detection, with a precision of $r=3.54^{\prime}$. We carried out a spectral analysis that adopts a power-law model. Leaving all the parameters free to vary, we obtain $N_{\mathrm{H}}=(1.62 \pm 0.09) \times 10^{22} \mathrm{~cm}^{-2}$, $\Gamma=(2.43 \pm 0.08)$, and $\chi_{r}^{2} /$ d.o.f. $=0.94 / 97$. The $2-10 \mathrm{keV}$

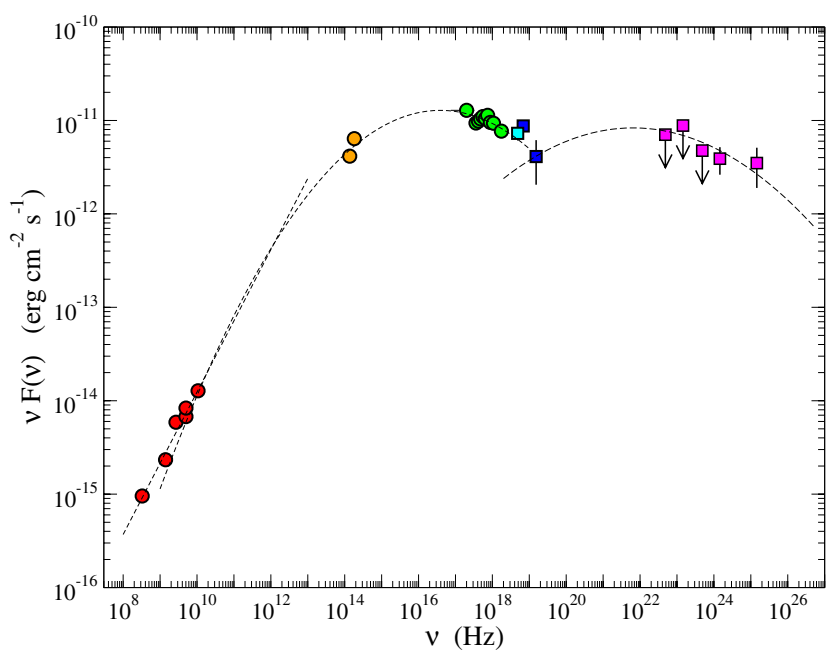

Fig. 5. The SED of 1FGL J2056.7+4938. From lower to higher frequencies we report radio data up to $22 \mathrm{GHz}$ (red circles) and data from 2MASS (orange circles), Swift-XRT (light green circles), Swift-BAT (cyan square), Integral-IBIS (blue squares) and Fermi-LAT (magenta squares). Data are fitted with a power-law model in the radio band; a log-parabolic model is used to emphasise the synchrotron and inverse Compton component of this high-energy synchrotron peak (HSP) BL Lac object.

flux is $1.19 \times 10^{-11} \mathrm{erg} \mathrm{cm}^{-2} \mathrm{~s}^{-1}$. We note that, unlike our result, Landi et al. (2010) report a value of the hydrogen column density $N_{\mathrm{H}}=(0.53 \pm 0.18) \times 10^{22} \mathrm{~cm}^{-2}$ lower than the Galactic contribution $\left(N_{\mathrm{H}}=1.0 \times 10^{22} \mathrm{~cm}^{-2}\right)$ quoted in the LAB Survey (Kalberla et al. 2005). We repeated the fit and fixed the $N_{\mathrm{H}}$ parameter to the Galactic one, but we do not find an acceptable variation in the $\chi^{2}$ value $\left(\chi_{r}^{2} /\right.$ d.o.f. $\left.=1.61 / 96\right) . \mathrm{A}$ broad band spectral fit of data from the radio to the $\mathrm{X}$ rays with a $\log$ parabola provides a curvature parameter $\beta_{\mathrm{S}}=0.07$ (Fig. 5). Therefore we repeated the fit of the X-ray spectrum with a log-parabolic model by fixing $\beta=0.07$ and also the hydrogen column density at a moderately higher value $\left(N_{\mathrm{H}}=\right.$ $1.2 \times 10^{22} \mathrm{~cm}^{-2}$ ) than the Galactic one; in this way, we obtain the acceptable result $\chi_{r}^{2} /$ d.o.f. $=1.14 / 94$.

The association between the radio and the soft X-ray emission can be considered firmly established by the short distance between the Swift and the XMM-Newton centroids, on the one hand, and the north-east component of $4 \mathrm{C}+49.35$, on the other. These are very close to the bright star SAO 50269 (RA = $20^{\mathrm{h}} 56^{\mathrm{m}} 41^{\mathrm{s}} .966 ;$ Dec $=+49^{\circ} 40^{\prime} 17^{\prime \prime} .55 ; R_{2}=8.39 \mathrm{mag}$ in USNOB1, Monet et al. 2003). The UVOT image in the UV-W2 filter does not show any evidence of sources in the proximity of the star, for which we obtain a magnitude $W 2=(13.12 \pm 0.05)$ mag. Both images acquired in the $U$ filter are saturated by the star. In a study of the low-latitude sample area in Cygnus of the ROSAT Galactic plane survey, Motch et al. (1997) associated the X-ray source RX J2056.6+4940 with SAO 50269 with a probability for a random association of about $10 \%$. The distance between the X-ray and the star position is $\sim 13^{\prime \prime}$ within the estimated ROSAT accuracy that is $\sim 25^{\prime \prime}$. The origin of the X-ray emission was related to an active corona surrounding this star, as for the $85 \%$ of sources with a PSPC count rate higher than $3 \times 10^{-2}$ cts $\mathrm{s}^{-1}$ belonging to their sample. Recently, Haakonsen \& Rutledge (2009) report a low probability of any association between 1RXS J205644.3+494011 and the star in their statistical cross-association of the ROSAT Bright Source Catalogue (Voges et al. 1999) and the 2MASS 
Point Source Catalogue (Cutri et al. 2003). As reported by Landi et al. (2010), the counterpart of this high-energy source is, with high probability, an object located at RA $=20^{\mathrm{h}} 56^{\mathrm{m}} 42^{\mathrm{s}} .719$ and Dec $=+49^{\circ} 40^{\prime} 06^{\prime \prime} 9$ at only 12'.9 from SAO 50269. It has been detected in the 2MASS infrared images retrieved from the NASA/IPAC infrared Science Archive. The magnitude in the $J$ filter is $13.69 \mathrm{mag}$ and is uncertain, whereas in the $H$ and $K$ filters they are $(14.30 \pm 0.10) \mathrm{mag}$ and $(13.74 \pm 0.08) \mathrm{mag}$, respectively. The emission of this object in the optical as well as in the UV band is most likely overwhelmed by that originating from the star. This source is separated from the $\gamma$-ray centroid by 1.5 .

Paredes et al. (2002) cited the ROSAT source 1RXS J205644.3+494011 in a search for microquasar candidates at low Galactic latitudes, which was obtained by means of a cross-identification between the ROSAT bright source catalogue (RBSC, Voges et al. 1999) and the NVSS catalogue. These authors gave a priority to sources for which the offset between the X-ray and the radio position was within the $1 \sigma$ RBSC position error; unfortunately, this was not the case for 1RXS J205644.3+494011. Recently, the hypothesis that the nature of this source might be extragalactic has been considered by several authors (Landi et al. 2010; Voss \& Ajello 2010; Stephen et al. 2010). We collected for the first time all the available data in the literature to build the SED of this object, which is reported in Fig. 5, to investigate the broad-band emission properties of 1FGL J2056.7+4938. We exclude the radio measurements reported by surveys with a low resolution to avoid the risk of including a spurious contribution from the SW component of $4 \mathrm{C}+49.35$. We estimate a radio spectral index $\alpha_{r} \sim 0.24$ from the fit of data up to $22 \mathrm{GHz}$ (Petrov et al. 2007), and this value of $\alpha_{r}$ is indeed typical of flat spectrum radio sources. An evaluation of correct fluxes both at optical and at infrared wavelengths is difficult not only for the near star but also for the high value of the reddening $E(B-V)=2.85 \mathrm{mag}$ (Schlegel et al. 1998), which may be uncertain for such a low value of the Galactic latitude. We fit the data from radio to $\mathrm{X}$-ray frequencies with a log-parabola and obtain the peak frequency $v_{\mathrm{S}}=3.86 \times 10^{+16} \mathrm{~Hz}$, the corresponding maximum of synchrotron emission $v_{\mathrm{S}} F\left(v_{\mathrm{S}}\right)=1.28 \times 10^{-11} \mathrm{erg} \mathrm{cm}^{-2} \mathrm{~s}^{-1}$ and the curvature parameter $\beta_{\mathrm{S}}=0.07$. In carrying out our fit, we force the log-parabola profile to agree with the power-law fit to the radio data and assume $E(B-V)=1 \mathrm{mag}$, consistently lower than the value quoted by Schlegel et al. (1998). The uncertain infrared measurement in the $\mathrm{J}$ filter was omitted. We also carried out a fit of high-energy data from the hard X-ray to the $\gamma$-ray band and obtain $v_{\mathrm{IC}}=6.46 \times 10^{+21} \mathrm{~Hz}$ with a corresponding $v_{\mathrm{IC}} F\left(v_{\mathrm{IC}}\right)=8.32 \times 10^{-12} \mathrm{erg} \mathrm{cm}^{-2} \mathrm{~s}^{-1}$ and a curvature parameter $\beta_{\mathrm{IC}}=0.04$.

More precise measurements are needed to characterise both emission components of this source. Nevertheless, the analysis of its broad-band properties, such as the radio spectral index, the low Compton dominance, and the synchrotron peak frequency higher than $10^{16} \mathrm{~Hz}$, support the classification of 1FGL J2056.7+4938 as a blazar, and in particular as an HSP BL Lac object, but only the analysis of the optical spectrum can confirm this interpretation.

\section{Conclusions}

We have reported the results of our analysis devoted to research of the sources that show high-energy emission in both the Swift-BAT and the Fermi-LAT telescopes, according to the data collected by LAT over the first 11 months of operation and by BAT over a much longer period of 54 months. As expected, we only found very few sources: only $7 \%$ of those included in the 1FGL catalogue are characterised by any significant emission $(\sigma>3)$ as shown by the 54-month BAT 15-150 keV map. The larger fraction of them include extragalactic objects, and the dominant part is represented by blazars. We investigated their redshift distribution in greater detail making a distinction among its subclasses. Comparison with the distribution of the whole population classified in the second edition of the BZCAT catalogue shows that they are mainly closer than average to the observer.

Driven by the detection from both the BAT and the LAT instruments, we focused on a couple of objects with very low Galactic latitude. Our detailed analysis of their broad-band SED supports the classification of these objects as blazars, and in particular as high synchrotron peaked (HSP) BL Lac Objects. Analyses of the same kind can be carried out with success for other sources that are expected in the near future with the release of the second Fermi-LAT point source catalogue, based on two years of $\gamma$-ray data, and also a new Palermo BAT catalogue from analysing six years of BAT data.

Acknowledgements. The authors are grateful to the referee for the suggestions and comments that helped to improve the manuscript. They acknowledge financial support by ASI/INAF through contract I/011/07/0. Part of this work is based on archival data, software, or online services provided by the ASI Science Data Center (ASDC) and by the SIMBAD database operated at the CDS, Strasbourg, France.

\section{References}

Abdo, A. A., Ackermann, M., Ajello, M., et al. 2010a, ApJS, 188, 405 Abdo, A. A., Ackermann, M., Ajello, M., et al. 2010b, ApJ, 715, 429 Abdo, A. A., Ackermann, M., Agudo, I., et al. 2010c, ApJ, 716, 30 Atwood, W. B., Abdo, A. A., Ackermann, M., et al. 2009, ApJ, 697, 1071 Barthelmy, S. D., Barbier, L. M., Cummings, J. R., et al. 2005, Space Sci. Rev., 120,143

Bikmaev, I. F., Burenin, R. A., Revnivtsev, M. G., et al. 2008, Astron. Lett., 34, 653

Bird, A. J., Bazzano, A., Bassani, L., et al. 2010, ApJS, 186, 1

Condon, J. J., Cotton, W. D., Greisen, E. W., et al. 1998, AJ, 115, 1693

Cusumano, G., La Parola, V., Segreto, A., et al. 2010a, A\&A, 510, 48

Cusumano, G., La Parola, V., Segreto, A., et al. 2010b, A\&A 524A, 64

Cutri, R. M., Skrutskie, M. F., Van Dyk, S., et al. 2003, IPAC/California Institute of Technology

Haakonsen, C. B., \& Rutledge, R. E. 2009, ApJS, 184, 138

Hill, J. E., Burrows, D. N., Nousek, J. A., et al. 2004, Proc. SPIE, 5165, 217

Kalberla, P. M. W., Burton, W. B., Hartmann, D., et al. 2005, A\&A, 440, 775

Krivonos, R., Revnivtsev, M., Lutovinov, A., et al. 2007, A\&A, 475, 775

Landau, R., Golisch, B., Jones, T. J., et al. 1986, ApJ, 308, 78

Landi, R., Bassani, L., Malizia, A., et al. 2010, MNRAS, 403, 945

Maselli, A., Cusumano, G., Massaro, E., et al. 2010, A\&A, 520, A47

Massaro, E., Perri, M., Giommi, P., \& Nesci, R. 2004a, A\&A, 413, 489

Massaro, E., Perri, M., Giommi, P., et al. 2004b, A\&A, 422, 103

Massaro, E., Giommi, P., Leto, C., et al. 2009, A\&A, 495, 691

Massaro, E., Giommi, P., Leto, C., et al. 2010 [arXiv: 1006.0922]

Monet, D. G., Levine, S. E., Canzian, B., et al. 2003, AJ, 125, 984

Motch, C., Guillout, P., Haberl, F., et al. 1997, A\&A, 318, 111

Paredes, J. M., Ribó, M., \& Martí, J. 2002, A\&A, 394, 193

Petrov, L., Hirota, T., Honma, M., et al. 2007, AJ, 133, 2487

Saxton, R. D., Read, A. M., Esquej, P., et al. 2008, A\&A, 480, 611

Schlegel, D. J., Finkbeiner, D. P., \& Davis, M. 1998, ApJ, 500, 525

Segreto, A., Cusumano, G., Ferrigno, C., et al. 2010, A\&A, 510, 47

Stephen, J. B., Bassani, L., Landi, R., et al. 2010, MNRAS, 408, 422

Telezhinsky, I., Eckert, D., Savchenko, V., et al. 2010, A\&A, 522, A68

Voges, W., Aschenbach, B., Boller, T., et al. 1999, A\&A, 349, 389

Voss, R., \& Ajello, M. 2010, ApJ, 721, 1843

Watson, M. G., Schrder, A. C., Fyfe, D., et al. 2009, A\&A, 493, 339

Warwick, R. S., Marshall, N., Fraser, G. W., et al. 1981, MNRAS, 197, 865 